\title{
An Adaptive Feature Extraction Algorithm for Classification of Seismocardiographic Signals
}

\author{
Amirtaha Taebi", Student Member, IEEE, Brian E. Solar, and Hansen A. Mansy, Member, IEEE \\ Biomedical Acoustics Research Laboratory, University of Central Florida, Orlando, FL 32816, USA \\ \{taebi@knights., bsolar@knights., hansen.mansy@\} ucf.edu
}

\begin{abstract}
This paper proposes a novel adaptive feature extraction algorithm for seismocardiographic (SCG) signals. The proposed algorithm divides the SCG signal into a number of bins, where the length of each bin is determined based on the signal change within that bin. For example, when the signal variation is steeper, the bins are shorter and vice versa. The proposed algorithm was used to extract features of the SCG signals recorded from 7 healthy individuals (Age: $29.4 \pm 4.5$ years) during different lung volume phases. The output of the feature extraction algorithm was fed into a support vector machines classifier to classify SCG events into two classes of high and low lung volume (HLV and LLV). The classification results were compared with currently available non-adaptive feature extraction methods for different number of bins. Results showed that the proposed algorithm led to a classification accuracy of $\sim 90 \%$. The proposed algorithm outperformed the non-adaptive algorithm, especially as the number of bins was reduced. For example, for 16 bins, $F_{1}$ score for the adaptive and non-adaptive methods were $0.91 \pm 0.05$ and $0.63 \pm 0.08$, respectively.
\end{abstract}

Keywords-Seismocardiography (SCG), adaptive feature extraction, classification, cardiorespiratory, support vector machine (SVM).

\section{INTRODUCTION}

$\mathrm{V}^{\mathrm{i}}$ ibrations created by cardiac activities (such as valve opening and closure, blood flow momentum changes, and myocardial contraction) can be measured noninvasively at the chest surface. Seismocardiography (SCG) is a technique that measures these vibrations using, for example, an accelerometer [1]-[3]. SCG might provide information that complements other cardiac monitoring methods such as echocardiography and electrocardiography [4]-[7]. Several algorithms and methods have been used to study SCG features with different levels of success. Understanding different characteristics of SCG signals may lead to a better understanding of heart function, as well as a more successful characterization of these signals. Better characterization and classification of SCG signals in health and diseased can provide possible methods for diagnosing and monitoring cardiac mechanical activities.

SCG signal morphology is affected by different factors, such as breathing phase, patient posture, sensor position, etc. Machine learning algorithms might be used to group SCG events into groups where each group contains similar events.
This grouping improves the quality of the signal ensemble averages, which allows more accurate estimation of SCG features. For example, it was suggested [8] that inspiratory and expiratory SCG events have different morphologies. Later studies [9]-[12] revealed that SCG signal morphology depended more on the lung volume rather than the direction of respiratory flow (i.e., inspiration vs. expiration).

This study proposes and tests a new feature extraction method that adaptively creates a feature vector. The proposed feature extraction algorithm was then used to classify SCG events into low and high lung volume (LLV and HLV) classes. The performance of the proposed method was compared to the available non-adaptive feature extraction methods [13]. These feature extraction algorithms are described in section II. Section III includes the instrumentations and experimental setup. Results are presented and discussed in sections IV and V, respectively, followed by conclusions in section VI.

\section{FEATURE EXTRACTION AlgORITHM}

The time-domain feature extraction method for SCG signals proposed by Zakeri [13] divides each SCG event into a fixed number of equal-width bins. It then uses the signal average within each bin as a feature. The grouped averages from the bins constitutes the feature vector, which will have a reduced dimensionality compared with the original signal. However, in this method, there may be bins that lack additional useful information (i.e., may have redundant values) and, hence, do not significantly improve the classification accuracy.

The feature extraction algorithm proposed in this paper aims at solving this potential issue by removing possible redundancies. In addition to dimensionality reduction, the proposed algorithm prioritizes the parts of the SCG signal that has more variations by assigning more bins to those parts.

The algorithm begins by specifying a threshold, which is a certain fraction of the signal's peak-to-peak amplitude. The threshold is chosen such that it, later, would result in a certain number of bins. In the next step the entire SCG event is considered as one bin and the signal standard deviation within the bin is calculated. When the standard deviation is above the specified threshold, the bin is divided into 2. For the new bins to approximately have equal lengths, it is preferable (but not necessary) that the SCG event contains power of 2 data points.

This study was supported by NIH R44HL099053. 
The standard deviation calculations are then repeated for the newly created bins, and more new bins will be created until every bin meets the threshold criteria. The outputs of this process are two vectors, where one vector contains the adaptively-spaced bins and the other contains the signal averages within each bin. The pseudo-code for the proposed algorithm is provided in Algorithm 1.

\section{HUMAN STUDIES}

\section{A. Participants}

The experimental protocol used in this study was approved by the institutional review board of the University of Central Florida, Orlando, FL. A total of 7 adult males participated in our study. The subjects provided their informed consent and reported no history of cardiovascular disease verbally. The age, height, and weight of the subjects were obtained and are reported in Table I.

\section{B. Experiments}

For breathing pattern and tidal volume consistency, the subjects were instructed on how to breathe. For this purpose, a ventilator (Model: 613, Harvard Apparatus, South Natick, MA)

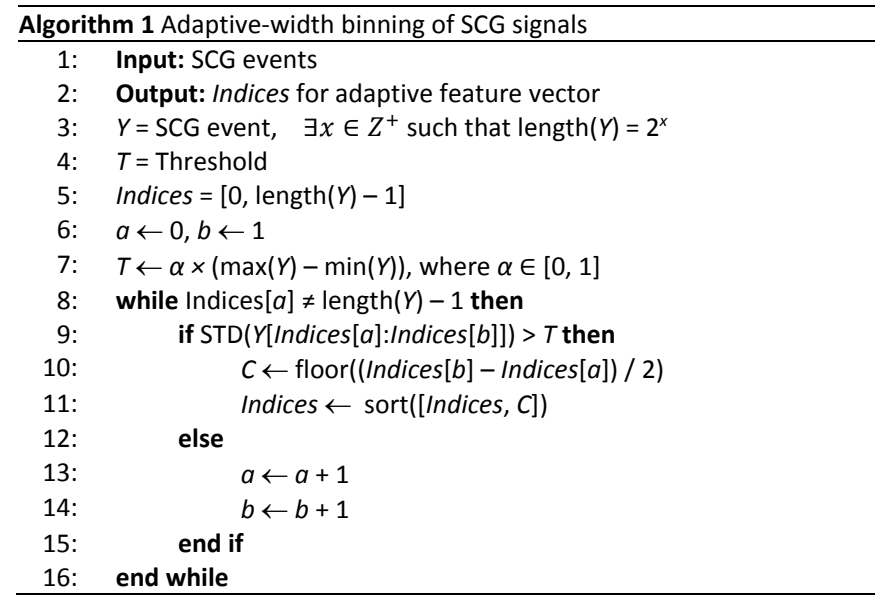

TABLE I OVERVIEW OF THE SUBJECTS' CHARACTERISTICS

\begin{tabular}{lc} 
& $($ MEAN \pm SD). \\
\hline Age (years) & $29.4 \pm 4.5$ \\
Height $(\mathrm{cm})$ & $173.1 \pm 9.8$ \\
Weight $(\mathrm{kg})$ & $82.2 \pm 18.3$ \\
Number of subjects & 7 \\
\hline
\end{tabular}

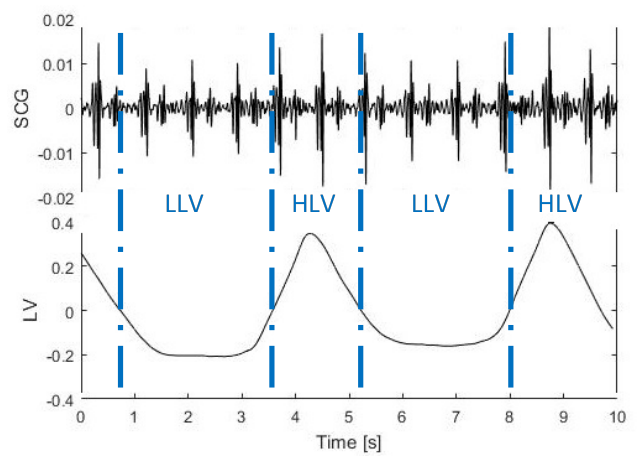

Fig. 1. (top) A 10-sec portion of the SCG signal, (bottom) Lung volume signal that was used to group SCG events into two groups of high and low was used to train subjects to breath with the same respiratory rate and inspiratory:expiratory (I:E) ratio. The respiratory rate and I:E ratio were set to 12 breath per minute and 1:3, respectively. The tidal volume (TV) for each subject was calculated in real time as time integral of the respiratory flow rate signal. During the experiment, TV was displayed on a computer screen and was about 10 to $15 \mathrm{~mL} / \mathrm{kg}$ for all subjects. The subjects rested on a folding bed with their chest tilted at 45 degrees and the signals of interest were recorded for 2 trials of 5 minutes each.

\section{Instrumentation}

A triaxial accelerometer (Model: 356A32, PCB Piezotronics, Depew, NY) measured all SCG signals. A signal conditioner (Model: 482C, PCB Piezotronics, Depew, NY) with a gain factor of 100 was used to amplify the accelerometer output. The accelerometer was attached with a double-sided medical-grade tape at the $4^{\text {th }}$ intercostal space and the left sternal border. The sensor location was chosen to attain a high signal-to-noise ratio [14], [15]. The accelerometer z-axis was perpendicular to the chest surface of the subject, while the yand $\mathrm{x}$-axes were aligned parallel to the mediolateral and axial directions, respectively. The respiratory flow rate was measured using a spirometer (Model: A-FH-300, iWorx Systems, Inc., Dover, NH), that was calibrated by the manufacturer. The expiration and inspiration produced negative and positive flow rate signal amplitudes, respectively. A Control Module (Model: IX-TA-220, iWorx Systems, Inc., Dover, $\mathrm{NH}$ ) was used to simultaneously acquire the voltage signal for respiratory flow rate, ECG, and SCG signals.

All above signals were simultaneously acquired with sampling rate of $10 \mathrm{kHz}$. To remove the remaining respiratory sound noise, the SCG signals were filtered using a low-pass filter with a cut-off of $100 \mathrm{~Hz}$ since lung sounds have significant energy above this cut-off frequency [16]. All signals were processed using Matlab (R2015b, The MathWorks, Inc., Natick, MA).

\section{SCG Event Pre-processing}

The SCG events in each signal were found using matched filtering with a template consisting of a previously identified SCG. The lung volume signal was then used to group SCG events into two groups of high and low lung volume. The SCG events that occurred during high and low lung volumes were called HLV and LLV SCG events, respectively (Fig. 1). The SCG events duration were chosen to contain 4096 (corresponding to $\sim 700$ milliseconds), which was sufficiently long to contain the SCG event. This was to ensure that all signals had equal length, and that the number of points for each event equals a power of 2 to best satisfy the algorithm's requirement.

\section{E. SCG Feature Extraction and Classification}

Feature extraction included dividing the SCG signal into bins $(16,32,64,128,256,512$, and 1024), calculating the mean of each bin, and treating this as the feature vector. This corresponds to feature vectors of length 16, 32, 64, 128, 256, 512, and 1024. This method was adopted from Zakeri [13]. The original method divided the signal into evenly spaced bins, and the new proposed method divides the signal into bins adaptively 
spaced using the introduced algorithm. However, when the proposed method is used, the location of the bins may be different for each SCG event, resulting in the possible transformation of each event to a different feature space. Hence, the adaptive method was instead applied to the ensemble average of all SCG events, and single set of bins was determined. The resulting bins were then used to create the feature vectors for the individual SCG events. After bins were finalized, the signal variability in each bin for the individual SCG events were checked to ensure that the variability does not exceed the threshold by more than $5 \%$.

A support vector machine (SVM) algorithm was used to classify the SCG events into two classes of LLV and HLV. The classifier used was the Radial Basis Function (RBF) SVM. The subject-specific training scenario was employed such that a different classification model was built for each individual subject. There was a total of 3986 samples (i.e. SCG events), $1813 \mathrm{HLV}$ and $2173 \mathrm{LLV}$. Per subject, there was an average of $259 \pm 48 \mathrm{HLV}, 310 \pm 42 \mathrm{LLV}$, and $569 \pm 76$ total samples.

The $k$-fold cross-validation method $(k=10)$ was used to evaluate the identification accuracy [17]. The accuracy was defined as the ratio of the number of correctly identified samples to the total number of samples in the test set. For each subject, the final accuracy of the model was obtained by averaging the 10 accuracies resulted from the $k$-fold cross-validation. The $F_{l}$ score was used as another evaluation metric [18]. The $F_{1}$ score was calculated as the harmonic mean of sensitivity and precision,

$$
F_{1}=2 \times(\text { sensitivity } \times \text { precision }) /(\text { sensitivity }+ \text { precision })
$$

where sensitivity and precision were defined as,

$$
\begin{aligned}
& \text { sensitivity }=T P /(T P+F N) \\
& \text { precision }=T P /(T P+F P)
\end{aligned}
$$

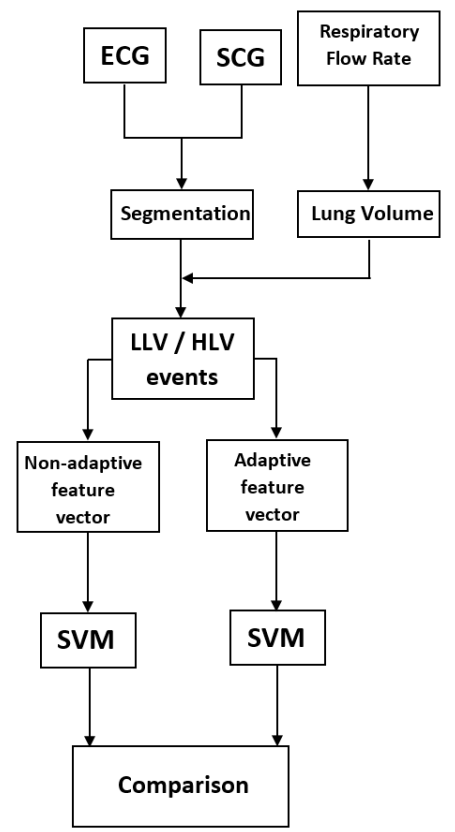

Fig. 2. Block diagram describing the signal processing and machine learning steps used in the current study. where $T P, F N$, and $F P$ were true positive, false negative, and false positive, respectively. The hyper parameters of the SVM models were chosen through an exhaustive grid search, with the goal of maximizing the 10 -fold cross-validation accuracy. The signal processing and machine learning steps for classification of LLV and HLV SCG events are shown in Fig. 2. The feature extraction and machine learning analysis was implemented with Python libraries Scikit-Learn [19] and SciPy/NumPy [20].

\section{RESUlts}

Fig. 3 shows the proposed adaptive algorithm (adaptivelyspaced bins) compared to the non-adaptive method (evenlyspaced bins) used in [13] for 16 bins.
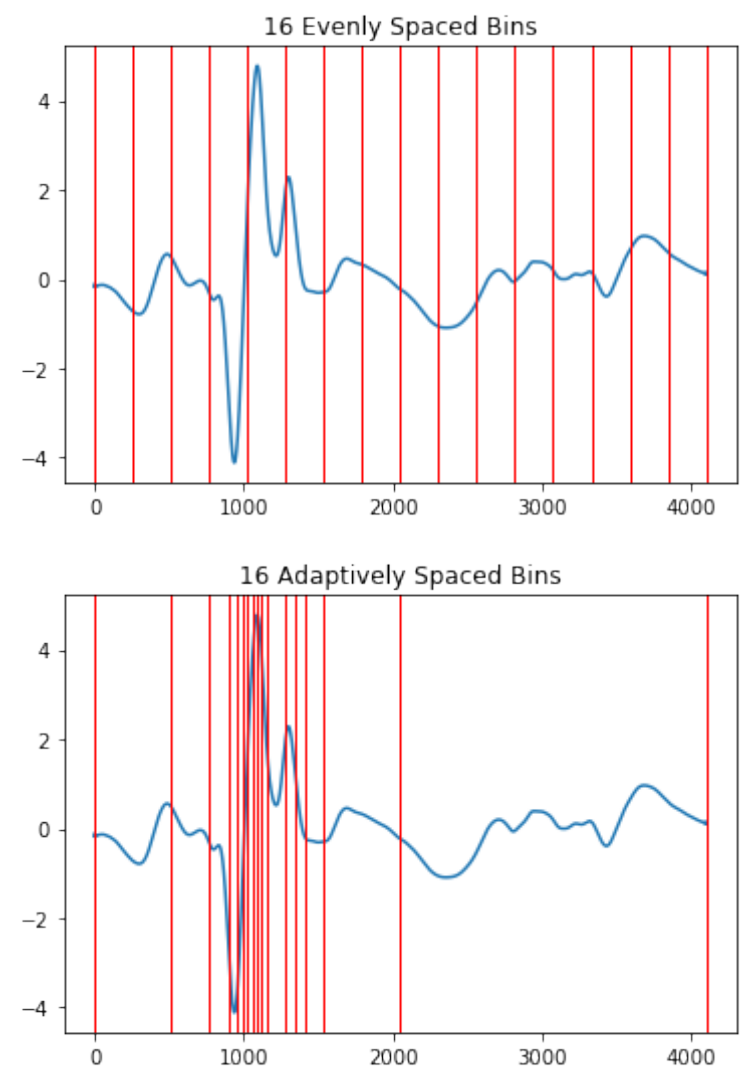

Fig. 3. (top) Equal-width bins configuration adopted from [13] vs (bottom) adaptive-width bins configuration proposed in this study for a feature vector length (i.e., number of bins) of 16.

TABLE II AVERAGE ACCURACIES AND $F_{l}$ SCORES OF SVM MODELS FOR DIFFERENT NUMBER OF BINS WHEN USING EQUAL-WIDTH (EW) AND ADAPTIVE-WIDTH (AW) BINS.

\begin{tabular}{ccccc}
\hline Bins & Acc. EW & Acc. AW & $\boldsymbol{F}_{1}$ EW & $\boldsymbol{F}_{1}$ AW \\
\hline $\mathbf{1 6}$ & $0.65 \pm 0.07$ & $0.90 \pm 0.05$ & $0.63 \pm 0.08$ & $0.91 \pm 0.05$ \\
$\mathbf{3 2}$ & $0.66 \pm 0.07$ & $0.91 \pm 0.04$ & $0.60 \pm 0.14$ & $0.92 \pm 0.04$ \\
$\mathbf{6 4}$ & $0.67 \pm 0.07$ & $0.91 \pm 0.04$ & $0.65 \pm 0.06$ & $0.93 \pm 0.04$ \\
$\mathbf{1 2 8}$ & $0.70 \pm 0.06$ & $0.92 \pm 0.04$ & $0.70 \pm 0.08$ & $0.92 \pm 0.04$ \\
$\mathbf{2 5 6}$ & $0.83 \pm 0.07$ & $0.92 \pm 0.04$ & $0.82 \pm 0.08$ & $0.92 \pm 0.04$ \\
$\mathbf{5 1 2}$ & $0.87 \pm 0.07$ & $0.92 \pm 0.04$ & $0.87 \pm 0.07$ & $0.91 \pm 0.05$ \\
$\mathbf{1 0 2 4}$ & $0.91 \pm 0.05$ & $0.92 \pm 0.04$ & $0.89 \pm 0.07$ & $0.91 \pm 0.05$ \\
\hline
\end{tabular}




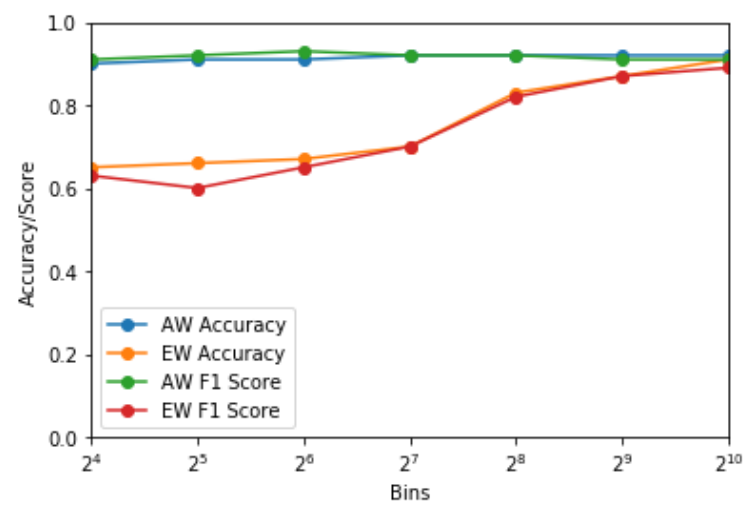

Fig. 4. Performance trend for SVM models using both AW and EW bins as the number of bins increase. The proposed adaptive feature extraction algorithm resulted in a higher accuracy and $F_{1}$ score consistently.

The classification of SCG events using the equal-width (EW) and adaptive-width (AW) bins began with a relatively small number of bins (16 bins). The performance analysis of the models was first done with these wider bins since it was less computationally expensive. The number of bins were then increased to find the number of bins at which the accuracy of the EW model reaches a plateau. This process is similar to mesh independency assessment in finite element analysis [21]-[30]. The identification accuracy for the SVM model for EW and AW bins feature vectors are listed in Table II for 7 different number of bins of $16,32,64,128,256,512$, and 1024. As the number of bins increased from 16 to 1024 , the accuracy of the EW increased from $65 \%$ to $91 \%$ while the accuracy of the AW seemed to be stay around $91 \%$ for all number of bins used. Similarly, the $F_{l}$ score of the model using EW raised from $63 \%$ to $89 \%$ by increasing the number of bins from 16 to 1024 . However, the $F_{1}$ scores of the model using adaptive feature vector were similar for different bin numbers. The accuracies and $F_{1}$ scores for the models using both the AW and EW bins converged as the number of bins increased, as displayed in Fig. 4. The accuracy and $F_{l}$ scores reached their maximum at a much smaller number of bins for the AW method, which can save calculation cost.

\section{Discussions}

\section{A. Algorithm Performance}

The performance of the SVM using AW or EW bins was comparable for high number of bins (1024 in the current study). The performance remained relatively constant from 16 to 1024 bins for AW bins, whereas reducing the number of bins significantly worsened the classification performance when EW bins were used. These results demonstrated that the AW bins resulted in higher accuracies at smaller number of bins, and therefore it was more computationally efficient. The improved performance of the adaptive feature extraction method might be due to higher density of bins around SCG1 (the relatively strong signal region around index 1000 of the SCG event shown in Fig. 3). SCG1 contains important physiological events such as aortic valve opening, mitral valve closure, isovolumic contraction, and rapid ejection [31], [32]. Therefore, focusing on this segment of the SCG event might increase the SVM classification accuracy.

\section{B. Limitations}

The primary limitation of the study is the small number of subjects and SCG event samples. More SCG events might result in a higher classification accuracy of the SVM models using both the AW and EW bins. Therefore, future studies need to enroll a larger number of subjects.

\section{CONCLUSIONS}

In this study, a SVM machine learning method was developed to identify the SCG events occurring during different phases of lung volume. To select features, an adaptive feature extraction algorithm was proposed. The proposed algorithm was found to be an efficient, reliable, and accurate approach to extract SCG features compared to the available feature extraction methods in literature. The feature extraction algorithm proposed in this study can be used in the analysis of other biomedical signals. In this study, the signal variability in temporal bins was measured by calculating the signal standard deviation. The proposed algorithm performance might be improved by employing other indicators of the signal complexity such as spectral entropy and average of the absolute values of the signal gradient (to measure the variation in slope).

\section{REFERENCES}

[1] A. Taebi and H. A. Mansy, "Time-Frequency Distribution of Seismocardiographic Signals: A Comparative Study," Bioengineering, vol. 4, no. 2, p. 32, 2017.

[2] J. Zanetti and D. Salerno, "Seismocardiography: A new technique for recording cardiac vibrations. Concept, method, and initial observations," J. Cardiovasc. Technol., vol. 9, no. 2, pp. 111-120, 1990.

[3] O. T. Inan, P. F. Migeotte, K. S. Park, M. Etemadi, K. Tavakolian, R. Casanella, J. Zanetti, J. Tank, I. Funtova, G. K. Prisk, and M. Di Rienzo, "Ballistocardiography and Seismocardiography: A Review of Recent Advances," IEEE J. Biomed. Heal. Informatics, vol. 19, no. 4, pp. 1414-1427, 2015.

[4] A. Taebi and H. A. Mansy, "Time-frequency Description of Vibrocardiographic Signals," in 38th Annual International Conference of the IEEE Engineering in Medicine and Biology Society, 2016.

[5] A. Taebi, R. H. Sandler, B. Kakavand, and H. A. Mansy, "Seismocardiographic Signal Timing with Myocardial Strain," in Signal Processing in Medicine and Biology Symposium (SPMB), 2017 IEEE, 2017, pp. 1-2.

[6] A. Taebi and H. A. Mansy, "Analysis of Seismocardiographic Signals Using Polynomial Chirplet Transform and Smoothed Pseudo Wigner-Ville Distribution," in Signal Processing in Medicine and Biology Symposium (SPMB), 2017 IEEE, 2017, pp. 1-6.

[7] A. Taebi and H. A. Mansy, "Effect of Noise on Time-frequency Analysis of Vibrocardiographic Signals," J. Bioeng. Biomed. Sci., vol. 6(202), p. 2, 2016.

[8] K. Tavakolian, A. Vaseghi, and B. Kaminska, "Improvement of ballistocardiogram processing by inclusion of respiration information.," Physiol. Meas., vol. 29, pp. 771-781, 2008.

[9] A. Taebi and H. A. Mansy, "Grouping Similar Seismocardiographic Signals Using Respiratory Information," in Signal Processing in Medicine and Biology Symposium (SPMB), 2017 IEEE, 2017, pp. 16.

[10] B. E. Solar, A. Taebi, and H. A. Mansy, "Classification of Seismocardiographic Cycles into Lung Volume Phases," in Signal Processing in Medicine and Biology Symposium (SPMB), 2017 IEEE, 2017, pp. 1-2.

[11] A. Taebi, A. J. Bomar, R. H. Sandler, and H. A. Mansy, "Heart Rate Monitoring During Different Lung Volume Phases Using Seismocardiography," in SoutheastCon 2018, IEEE, 2018, pp. 1-5.

[12] A. Taebi, "Characterization, Classification, and Genesis of 
Seismocardiographic Signals," University of Central Florida, 2018.

[13] V. Zakeri, A. Akhbardeh, N. Alamdari, R. Fazel-Rezai, M. Paukkunen, and K. Tavakolian, "Analyzing Seismocardiogram Cycles to Identify the Respiratory Phases," IEEE Trans. Biomed. Eng., vol. 64, no. 8, pp. 1786-1792, Aug. 2017.

[14] A. Taebi and H. A. Mansy, "Time-frequency Analysis of Vibrocardiographic Signals," in 2015 BMES Annual Meeting, 2015.

[15] A. Taebi and H. A. Mansy, "Noise Cancellation from Vibrocardiographic Signals Based on the Ensemble Empirical Mode Decomposition," J. Appl. Biotechnol. Bioeng., vol. 2, no. 2, p. 00024, 2017.

[16] H. A. Mansy, T. J. Royston, R. A. Balk, and R. H. Sandler, "Pneumothorax detection using computerised analysis of breath sounds.," Med. Biol. Eng. Comput., vol. 40, no. 5, pp. 526-532, 2002.

[17] J. D. Rodríguez, A. Pérez, and J. A. Lozano, "Sensitivity Analysis of k-Fold Cross Validation in Prediction Error Estimation," IEEE Trans. Pattern Anal. Mach. Intell., vol. 32, no. 3, pp. 569-575, 2010.

[18] D. M. W. Powers, "Evaluation: From Precision, Recall and FMeasure To Roc, Informedness, Markedness \& Correlation," $J$. Mach. Learn. Technol., vol. 2, no. 1, pp. 37-63, 2011.

[19] F. Pedregosa and G. Varoquaux, Scikit-learn: Machine learning in Python, vol. 12. 2011.

[20] S. Van Der Walt, S. C. Colbert, and G. Varoquaux, "The NumPy array: A structure for efficient numerical computation," Comput. Sci. Eng., vol. 13, no. 2, pp. 22-30, 2011.

[21] F. Khalili, P. P. T. Gamage, and H. A. Mansy, "Verification of Turbulence Models for Flow in a Constricted Pipe at Low Reynolds Number," in 3rd Thermal and Fluids Engineering Conference (TFEC), 2018, pp. 1-10.

[22] F. Khalili, P. P. T. Gamage, and H. A. Mansy, "Prediction of Turbulent Shear Stresses through Dysfunctional Bileaflet Mechanical Heart Valves using Computational Fluid Dynamics," in 3rd Thermal and Fluids Engineering Conference (TFEC), 2018, pp. 1-9.

[23] F. Khalili, P. P. T. Gamage, and H. A. Mansy, "The Influence of the Aortic Root Geometry on Flow Characteristics of a Bileaflet Mechanical Heart Valve," 3rd Therm. Fluids Eng. Conf., 2018.

[24] P. P. T. Gamage, F. Khalili, and H. A. Mansy, "Numerical Modeling of Pulse Wave Propagation in a Stenosed Artery using Two-Way Coupled Fluid Structure Interaction (FSI)," in 3rd Thermal and Fluids Engineering Conference (TFEC), 2018.

[25] F. Khalili, P. P. T. Gamage, and H. A. Mansy, "Hemodynamics of a Bileaflet Mechanical Heart Valve with Different Levels of Dysfunction," J Appl Biotechnol Bioeng, vol. 2, no. 5, p. 00044, 2017.

[26] F. Khalili and H. A. Mansy, "Blood Flow through a Dysfunctional Mechanical Heart Valve," in 38th Annu Int Conf IEEE Eng Med Biol Soc, 2016.

[27] F. Khalili, "Fluid Dynamics Modeling and Sound Analysis of a Bileaflet Mechanical Heart Valve," University of Central Florida, 2018.

[28] A. Sharifi, A. Salari, A. Taebi, H. Niazmand, and M. J. Niazmand, "Flow patterns and wall shear stress distribution in human vertebrobasilar system: A computational study to investigate smoking effects on atherosclerotic stenosis at different ages," in ASME International Mechanical Engineering Congress and Exposition, Proceedings (IMECE), 2017, vol. 3.

[29] P. Thibbotuwawa Gamage, "Modeling of flow generated sound in a constricted duct at low Mach number flow," 2017.

[30] A. Taebi, F. Khalili, and A. Taebi, "Buckling analysis of a functionally graded implant model for treatment of bone fractures: A numerical study," in ASME International Mechanical Engineering Congress and Exposition, Proceedings (IMECE), 2017, vol. 3.

[31] D. M. Salerno and J. Zanetti, "Seismocardiography for monitoring changes in left ventricular function during ischemia," Chest, vol. 100, no. 4, 1991.

[32] R. S. Crow, P. Hannan, D. Jacobs, L. Hedquist, and D. M. Salerno, "Relationship between Seismocardiogram and Echocardiogram for
Events in the Cardiac Cycle," Am. J. Noninvasive Cardiol., vol. 8, no. 1, pp. 39-46, 1994. 